\title{
The PyPIF5-PymiR156a-PySPL9-PyMYB114/ MYB10 module regulates light-induced anthocyanin biosynthesis in red pear
}

Hainan Liu ${ }^{1,2+}$, Qun Shu ${ }^{3+}$, Kui Lin-Wang ${ }^{4}$, Andrew C. Allan ${ }^{4,5}$, Richard V. Espley ${ }^{4}$, Jun Su ${ }^{3}$, Maosong Pei ${ }^{1,2}$ and Jun $\mathrm{Wu}^{1 *}$

\begin{abstract}
Some cultivars of pear (Pyrus L.) show attractive red fruit skin due to anthocyanin accumulation. This pigmentation can be affected by environmental conditions, especially light. To explore the light-induced regulation network for anthocyanin biosynthesis and fruit coloration in pear, small RNA libraries and mRNA libraries from fruit skins of 'Yunhongyihao' pear were constructed to compare the difference between bagging and debagging treatments. Analysis of RNA-seq of fruit skins with limited light (bagged) and exposed to light (debagged), showed that PyPIF5 was down-regulated after bag removal. PymiR156a was also differentially expressed between bagged and debagged fruit skins. We found that PyPIF5 negatively regulated PymiR156a expression in bagged fruits by directly binding to the G-box motif in its promoter. In addition, PymiR156a overexpression promoted anthocyanin accumulation in both pear skin and apple calli. We confirmed that PymiR156a mediated the cleavage of PySPL9, and that the target PySPL9 protein could form heterodimers with two key anthocyanin regulators (PyMYB114/PyMYB10). We proposed a new module of PyPIF5-PymiR156a-PySPL9-PyMYB114/MYB10. When the bagged fruits were reexposed to light, PyPIF5 was down-regulated and its inhibitory effect on PymiR156a was weakened, which leads to degradation of the target PySPL, thus eliminating the blocking effect of PySPL on the formation of the regulatory MYB complexes. Ultimately, this promotes anthocyanin biosynthesis in pear skin.
\end{abstract}

Keywords: Red-skinned pear, Anthocyanin, PyPIF5, PymiR156a, PySPL9, PyMYB114/MYB10

Gene \& Accession Numbers: PyMYB10 (Pbr016663.1); PyMYB114 (Pbr042924.1); PyPIF5 (Pbr029607.1); PySPL9 (Pbr019232.1); PybHLH3 (Pbr017379.1); PyERF3 (Pbr030451.1).

\section{Core}

A novel regulatory module PyPIF5-PymiR156a-PySPL9PyMYB114/MYB10 underlying the light-induced anthocyanin biosynthesis in red pear is unraveled. Light down-regulates PyPIF5, which releases PymiR156a from PyPIF5's inhibition and allows PymiR156a to degrade its

\footnotetext{
* Correspondence: wujun@njau.edu.cn

${ }^{+}$Hainan Liu and Qun Shu contributed equally to this work.

${ }^{1}$ College of Horticulture, State Key Laboratory of Crop Genetics and

Germplasm Enhancement, Nanjing Agricultural University, Nanjing 210095, China

Full list of author information is available at the end of the article
}

target PySPL transcripts, promoting the formation of the PyMYB114/MYB10 complexes that increase the anthocyanin biosynthesis.

\section{Introduction}

Pear (Pyrus L.) is an important temperate deciduous fruit crop. Compared to pears with green or brown (russetted) skins, red skinned varieties are popular but relatively scarce. Pigmentation of most fruits begins at the middle or late stages of fruit development, with coloration partly determined by environmental influences (Yang et al. 2013), especially light. Light is generally

C C The Author(s). 2021 Open Access This article is licensed under a Creative Commons Attribution 4.0 International License, which permits use, sharing, adaptation, distribution and reproduction in any medium or format, as long as you give appropriate credit to the original author(s) and the source, provide a link to the Creative Commons licence, and indicate if changes were made. The images or other third party material in this article are included in the article's Creative Commons licence, unless indicated otherwise in a credit line to the material. If material is not included in the article's Creative Commons licence and your intended use is not permitted by statutory regulation or exceeds the permitted use, you will need to obtain permission directly from the copyright holder. To view a copy of this licence, visit http://creativecommons.org/licenses/by/4.0/. The Creative Commons Public Domain Dedication waiver (http://creativecommons.org/publicdomain/zero/1.0/) applies to the data made available in this article, unless otherwise stated in a credit line to the data. 
necessary for anthocyanin biosynthesis and organ coloration in various plants (Azuma et al. 2012; Feng et al. 2013; Jiang et al. 2017). In previous studies, MYB (v-myb myeloblastosis viral oncogene homolog), bHLH (Basic Helix-Loop-Helix) and WDR (WD40-repeat) were found to be the key transcription factors (TFs) that regulate anthocyanin biosynthesis, binding to the promoters of genes that encode enzymes of the anthocyanin biosynthetic pathway Espley et al. 2007, Toledo-Ortiz et al. 2003 (Xu et al. 2015). These TFs form the MBW transcription complex, such as found in Arabidopsis (Xu et al. 2013), Petunia (Quattrocchio et al. 1993), and apple (An et al. 2012; Liu et al. 2017). Besides the MBW complex, a new PybHLH3-PyMYB114-PyERF3 transcription complex was found in pear (Yao et al. 2017). Other regulatory factors have also been reported, such as WRKY (An et al. 2019; Li et al. 2020), NAC (Zhou et al. 2015) and HB (HD-Zip) (Jiang et al. 2017). In addition, some light-responsive TFs have been found to affect anthocyanin biosynthesis by regulating genes that encode enzymes of the pathway, such as HY5 (An et al. 2017), BBX (Bai et al. 2019; Xu et al. 2016) and bZIPa (Liu et al. 2019).

While anthocyanin biosynthesis is regulated at the transcriptional level, the expressed genes are also affected by RNA processing (post-transcriptional regulation) (Cui et al. 2014; Gou et al. 2011; Yang et al. 2019). MicroRNA (miRNA) plays an important role in posttranscriptional gene regulation. Multiple light response elements were found in miRNAs (Gou et al. 2011) and increasing studies have shown that miRNAs, along with their downstream targets, play important roles in development, growth and other important life processes in plants (Cui et al. 2020; Guo et al. 2019; Miao et al. 2019; $\mathrm{Yu}$ et al. 2019). Some of these miRNA-mediated processes overlap with anthocyanin biosynthesis, such as miR156 targeted AtSPL9 which acts as a negative regulator of anthocyanin biosynthesis in Arabidopsis (Gou et al. 2011). Stress-induced anthocyanin accumulation in Arabidopsis is regulated by the miR156-SPLs-DFR pathway (Cui et al. 2014). MiR156a also plays an important role during light-induced anthocyanin biosynthesis in apple skin, and was shown by using lncRNA MLNC3.2 and MLNC4.6 as endogenous target mimics, to prevent cleavage effects of miR156a on SPL2-like and SPL33 mRNA (Yang et al. 2019). These results suggested that the regulatory pathway of miR156 was different across diverse species. To date, the miR156 regulatory pathway in red pear is unknown. Furthermore, other miRNA family members and their corresponding targets are involved in the regulation of anthocyanin biosynthesis, such as miR399d/PHO2 (Peng et al. 2020), miR858/ MYB (Jia et al. 2015) and miR828/MYB (Yang et al. 2013). Research tends to focus on the relationship of
miRNA-target genes and how miRNAs exert function through these genes. There are fewer studies on miRNA regulatory factors. In regard to the process of lightinduced anthocyanin biosynthesis, the upstream regulatory mechanism of anthocyanin associated-miRNAs has not yet been reported.

In this study, we compared the transcriptome and small RNA data of bagged and debagged pear fruit skins, providing new data for the exploration of the miRNA regulated light-induced anthocyanin biosynthesis pathway and the discovery of the upstream regulator of miRNAs. Our study attempts to establish a regulation model of upstream regulator-miRNA-target-transcription factor of structural genes under light-induced condition.

\section{Results}

Identification of DE-miRNAs and DEGs

Anthocyanin accumulated rapidly in the pear fruit skin at 4 (D1), 8 (D2), and 10 (D3) days after bag removal, but no anthocyanin accumulation was observed in the corresponding days with bagged fruits (B1, B2, B3) (Fig. 1A). To examine miRNAs levels, we performed small RNAs (sRNAs) sequencing from pear skins of bagged (B1, B2, B3) and debagged (D1, D2, D3) fruits. After removing low-quality reads and adapter sequences, clean reads were used for identification of miRNAs. A total of 34 conserved miRNA families were identified. In addition, there were 110,43 and 34 differentially expressed (DE)-miRNAs in bagged (B1, B2, B3) and debagged fruit skins (D1, D2, D3) (Fig. 1B). A total of 55, 22 and 20 DE-miRNAs were upregulated and 55, 21 and 14 were downregulated in comparisons of D1/B1, D2/B2 and D3/B3 (Fig. 1C).

To investigate the expression profile of miRNAstargeted genes, RNA sequence analysis was performed using corresponding samples. An average of 9 billion clean bases was generated for each sample. There were 4775, 6670, 6936 differentially expressed genes (DEGs) identified in bagged and debagged fruit skins (Fig. 1B) at the three time points, with 2957, 3529 and 3596 upregulated and 1818, 3141 and 3340 downregulated in D1/B1, D2/B2 and D3/B3 (Fig. 1C). Furthermore, a cluster analysis of DE-miRNAs and DEGs showed that bagging and debagging treatments caused dramatic change in expression levels of miRNAs and mRNAs, and bagged and debagged fruits can be well divided according to their expression profiles (Fig. 1D).

Based on the functional enrichment analysis for predicted targets and DEGs (Fig. S1A-D), 10 conserved DEmiRNAs from 6 miRNA families (including miR858, miR408, miR399, miR164, miR172 and miR156) and 628 DEGs from 23 family (such as COP, PIF, bZIP, MYB, $S P L, N A C$, etc.) were selected as candidate regulators involved in light-induced anthocyanin regulation. In 


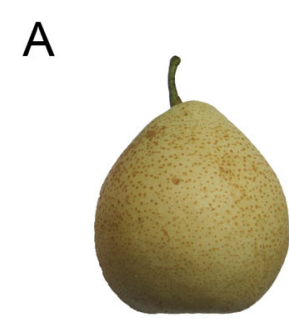

B1

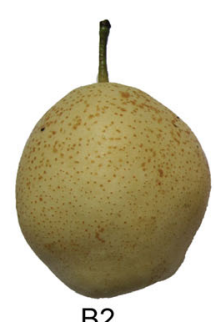

B2

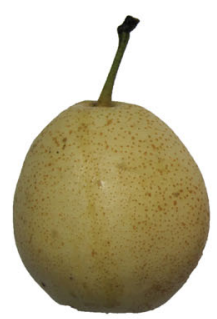

B3

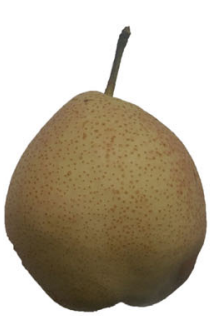

D1

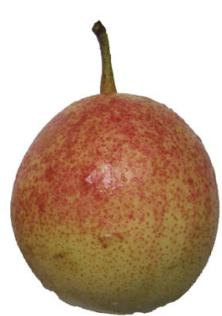

D2

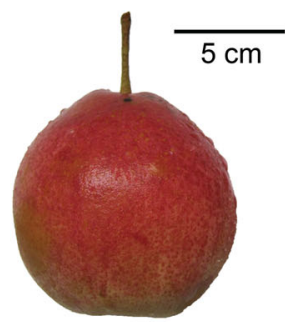

D3
B

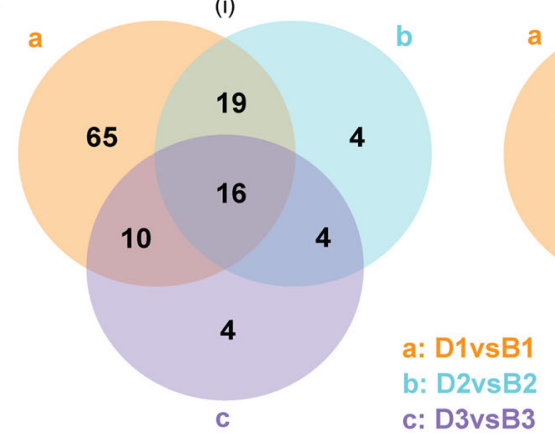

D

(i)

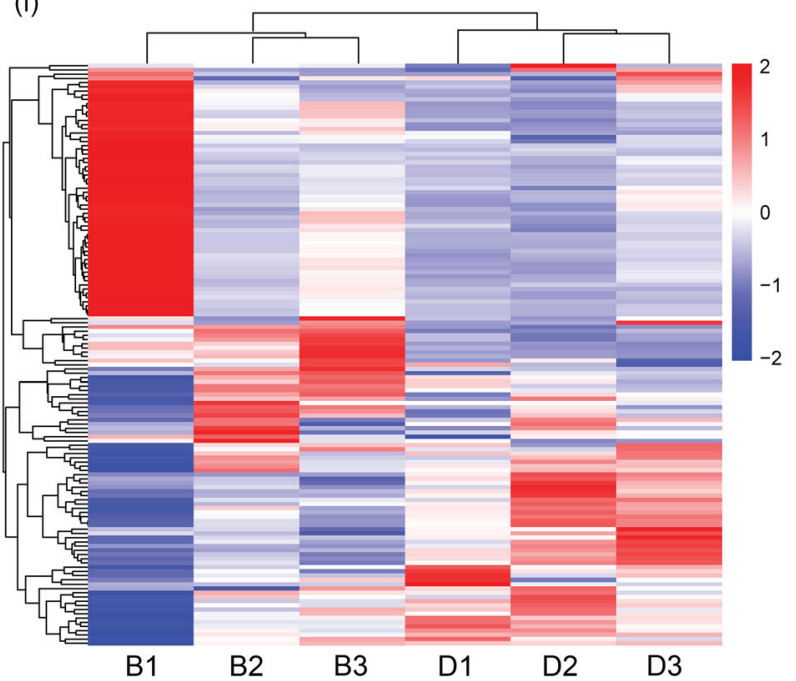

(ii)

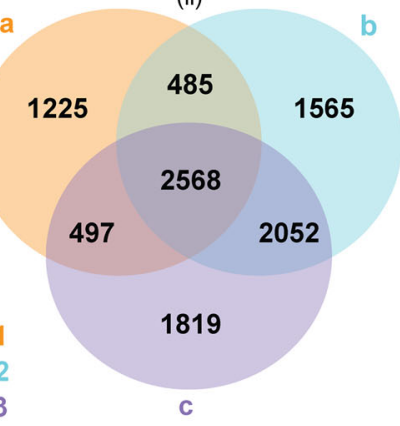

C

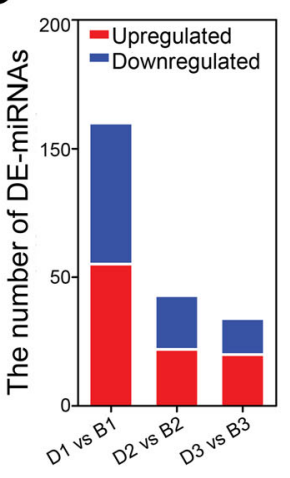

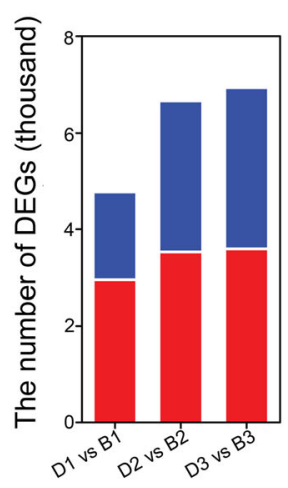

(ii)

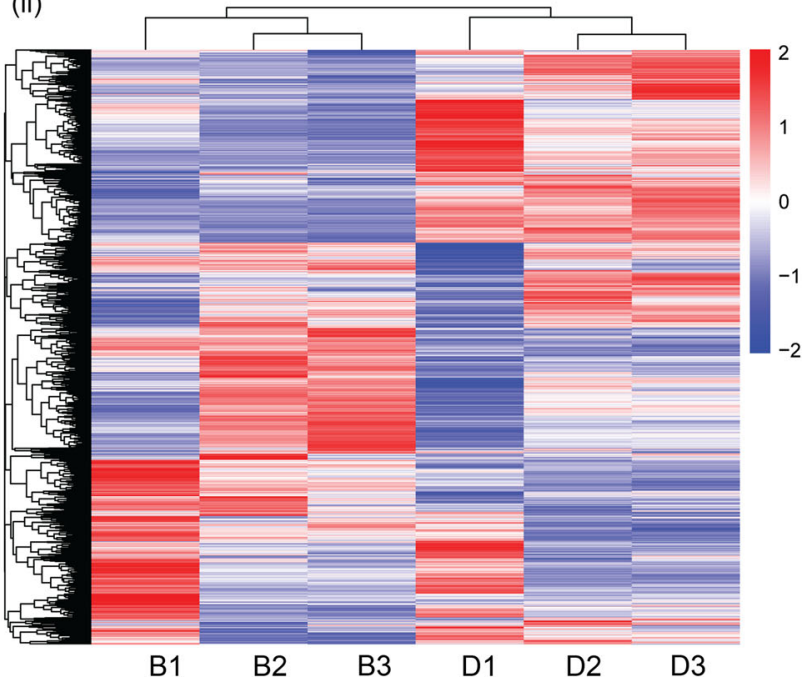

B1

B2 B

Fig. 1 Phenotype of "Yunhongyihao" and sequencing of sRNAs and RNA in bagged and debagged fruits. A Phenotype of "Yunhongyihao" at 4, 8, and 10 days after bag removal for bagged (B1, B2, B3) and debagged (D1, D2, D3) fruits. B Venn diagram showing the number of DE-miRNAs (i) and DEGs (ii). C The number of upregulated and downregulated DE-miRNAs and DEGs. D Cluster analysis of the expression levels of DE-miRNAs (i) and DEGs (ii)

addition, 329 predicted target genes of candidate DEmiRNAs were overlapped with genes differentially expressed in at least one comparison (Fig. S1E). The predicted targets of PymiR156a had the highest overlap ratio with DEGs, which indicated that PymiR156a had significant regulation on target genes, and may play an important regulatory role during light-induced anthocyanin biosynthesis.

Histochemical analysis of $\beta$-glucuronidase (GUS) expression driven by PymiR156a promoter indicated that the PymiR156a promoter had the ability of initiating gene expression of GUS reporter gene (Fig. S2). Furthermore, we analyzed promoter region of pre-miR156a (the genomic region 3000-bp upstream of PymiR156a precursor) using the PlantCARE online software (http:// bioinformatics.psb.ugent.be/webtools/plantcare/html/). There were 17 light-responsive elements in the premiR156a promoter, including one AE-box, three Box 4, five G-boxes, two GATA-motifs, two GT1-motifs, one Ibox, one LAMP-element, one sp1 element, and one 
TCCC-motif (Supplementary Table S1). Among these elements, multiple typical PIF-binding sites (G-box motif) were found in the PymiR156a promoter. Interestingly, we found a phytochrome-interacting transcription factor, PyPIF5, was down-regulated by bag removal, and the expression of PyPIF5 and PymiR156a showed a negative correlation, suggesting that there may be a regulatory relationship between them.

\section{PyPIF5 suppresses PymiR156a expression by binding to a G-box in its promoter}

PIFs belong to the basic helix-loop-helix (bHLH) family with a typical highly conserved APB domain [active phytochrome B (phyB) binding] (Fig. S3). We found PyPIF5 was down-regulated in debagged fruits (Fig. 2A). Furthermore, multiple PIF-binding sites were found in the PymiR156a promoter (Supplementary Table S1), which suggested that the down-regulated PyPIF5 may be involved in expression suppression of PymiR156a. Therefore, we focus on the regulatory relationship of PyPIF5 to PymiR156a. For its functional verification, we suppressed and overexpressed PyPIF5 in "Zaosu" pear skins by using agrobacterium-mediated transient expression method, and observed that the suppression of PyPIF5 could produce the red patches on pear skins, while no visible red patches in overexpression treatment or empty vector control (Fig. 2B). The qRT-PCR analysis of the infiltrated pear skin patches showed upregulation of PymiR156a in PyPIF5 overexpression patches and downregulation of PymiR156a in PyPIF5 suppression patches, and the expression pattern of PySPL9 was negatively correlated with that of PymiR156a (Fig. 2C). These above results show that alteration of PyPIF5 expression affected anthocyanin production with associated changes of PymiR156a and PySPL9. Dual-luciferase assays suggested that the co-expression of PyPIF5 strongly suppressed the $L U C$ reporter gene activities when driven by PymiR156a promoter, indicating the inhibitory effect of PyPIF5 on PymiR156a promoter (Fig. 2D).

The regulatory relationship of PyPIF5 to PymiR156a was further tested by Electrophoretic mobility shift assay (EMSA). We expressed and purified recombinant PyPIF5-His fusion protein and prepared PymiR156a promoter probe containing G-box motif (and its corresponding mutated sequence) (Fig. S4), to test whether PyPIF5 protein could directly interact with the PymiR156a promoter in vitro. EMSA demonstrated that PyPIF5 bound to the oligonucleotide sequence of the Gbox in PymiR156a promoter, and competitive reactions (with cold probe competitor and mutated probe competitor) showed that the binding reaction was specific. PyPIF5 directly bound to the biotin labeled probes. This binding activity was gradually reduced upon the adding of unlabeled cold probe. Furthermore, mutant versions of probes with mutations destroying the G-box motif showed no binding of PyPIF5. All together, these results demonstrated that PyPIF5 directly binds to the PymiR156a promoter, which is consistent with the observation that PyPIF5 regulates PymiR156a expression abundance (Fig. 2E). Thus, we concluded that PyPIF5 protein could specifically bind to G-box motif in the PymiR156a promoter and repress its expression.

\section{Target gene prediction and genome-wide analysis of miR156 targets}

Compared with bagged fruits, the expression of PymiR156a significantly increased in debagged fruits (Fig. 3A). Squamosa promoter binding protein-like (SPL) family members were predicted as PymiR156atargets (Fig. 3B) by using the psRNATarget server (Dai and Zhao 2011). In total, 19 PySPL genes were identified in the pear genome. A phylogenetic tree was constructed using SPL amino acid sequences from pear, apple and Arabidopsis (by using neighborjoining algorithm). These SPL proteins were clustered into seven groups, and PySPLs (marked with black solid dots) were distributed in seven cluster groups (Fig. 3C). All PySPL members have a conserved SBP domain, containing two zinc finger-like structures $(\mathrm{Zn}-1$ and -2) and a highly conserved nuclear localization signal (NLS) (Fig. 3D).

In contrast to the PymiR156a expression pattern, we observed a significant decrease in the expression levels of PySPL2, 5, 7, 9, 10, 13, 16, 17 and PySPL18 at least in one comparison (D1/B1, D2/B3, or D3/B3) (Fig. 3E). The predicted protein sequence of PySPL9 has high sequence similarity to A. thaliana AtSPL9 (shown in Fig. 3C), which was reported to act as a negative regulator of anthocyanin biosynthesis (Gou et al. 2011). To examine the subcellular localization of PySPL proteins, a PySPL9-GFP fusion construct was generated and transferred into tobacco by Agrobacterium-mediated transformation. Green fluorescence signals were observed specifically in the nucleus by the colocalization with DAPI (4',6-Diamidino-2-Phenylindole) staining. The result confirms that PySPL9 protein is a nuclear protein (Fig. 3F), which is consistent with the identification of an NLS in the SBP domain.

Effector vectors were constructed to express the premiR156a (Ox-miR156a) and short tandem target mimicmiR156a (STTM-miR156a) structure, and a reporter vector containing LUC (firefly luciferase) gene fused to PymiR156a target site sequences (Target site-SPLs). After co-infiltration into Nicotiana benthamiana leaves, the target site-SPLs and STTM-miR156a effector vector showed a significantly higher LUC/REN ratio than that of Ox-miR156a (Fig. 3G). These results show that the predicted target sites in pear SPL mRNA can be identified and cleaved by PymiR156a. 

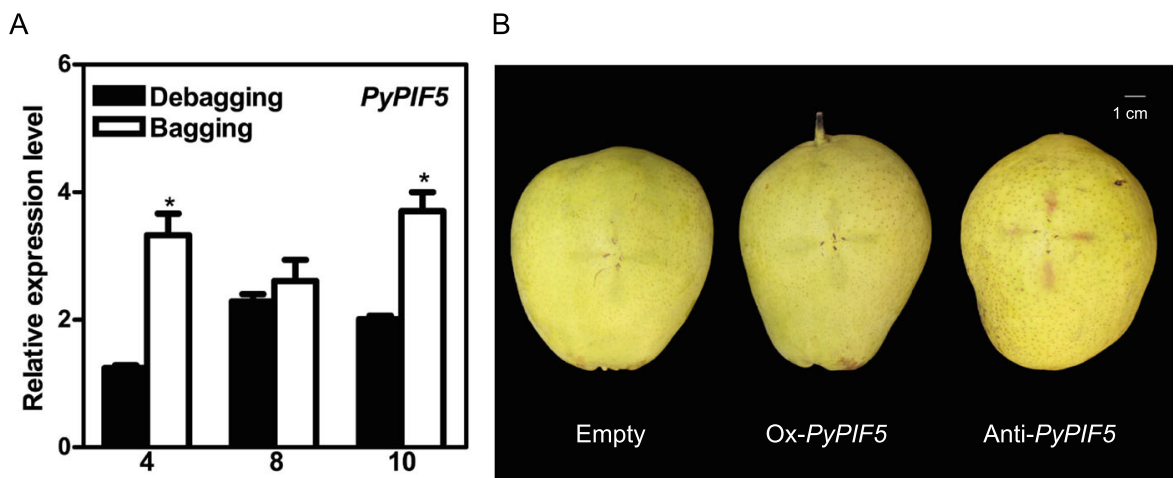

Days after bag removal

C
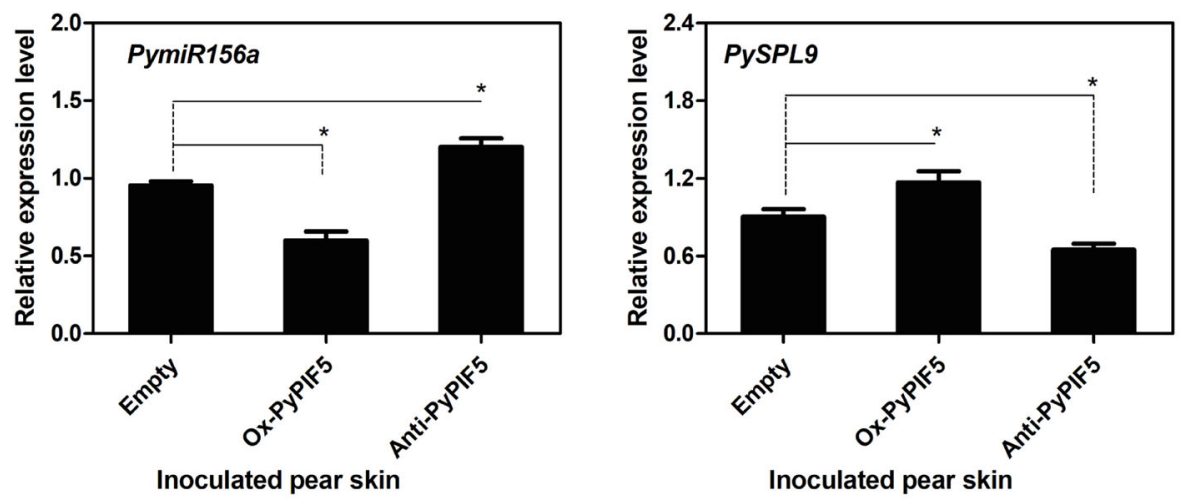

D
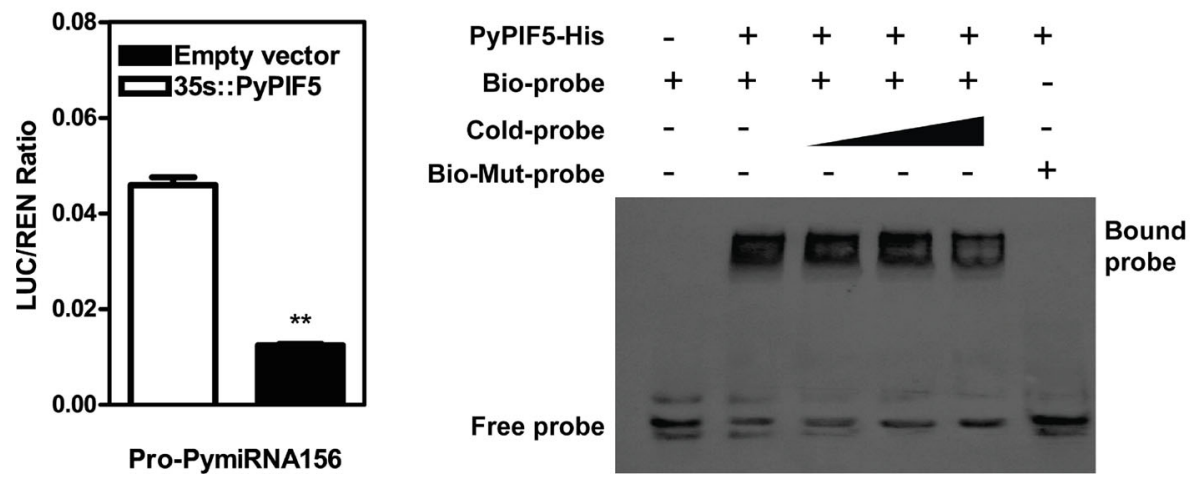

Fig. 2 PyPIF5 protein suppresses PymiR156a expression by binding G-box in promoter. A The expression profile of PyPIF5. B Overexpression and suppression of PyPIF5. C Expression pattern analysis PymiR156a and PySPL9 in inoculated pear. D Dual-luciferase assay for the transcriptional activation of PyPIF5. E Electrophoretic mobility shift assay for examining the DNA-binding property of PyPIF5. As the progressively increase of Cold probe concentration, the accumulation of Free probe increases. The addition of mutant probes further confirmed that PyPIF5 can specifically bind to the G-box sequence within PymiR156a promoter

\section{Overexpression of PymiR156a promotes anthocyanin accumulation}

To investigate the function of PymiR156a, we transformed overexpression (Ox-miR156a) and suppression (STTM-miR156a) constructs (also fused to Green fluorescent protein reporter, GFP) into apple calli through Agrobacterium-mediated genetic transformation according to An et al. (2017). The PymiR156a overexpression calli turned red, while WT control and PymiR156a suppression calli did not (Fig. 4A). GFP detection in apple calli showed that the GFP was expressed both in overexpression and suppression calli (Fig. 4A), which verifies successful transformation of the apple calli. Transient fruit transformation was also performed to the pear cultivar "Zaosu", and the same phenotypes were also observed (Fig. 4B). We also observed anthocyanin 

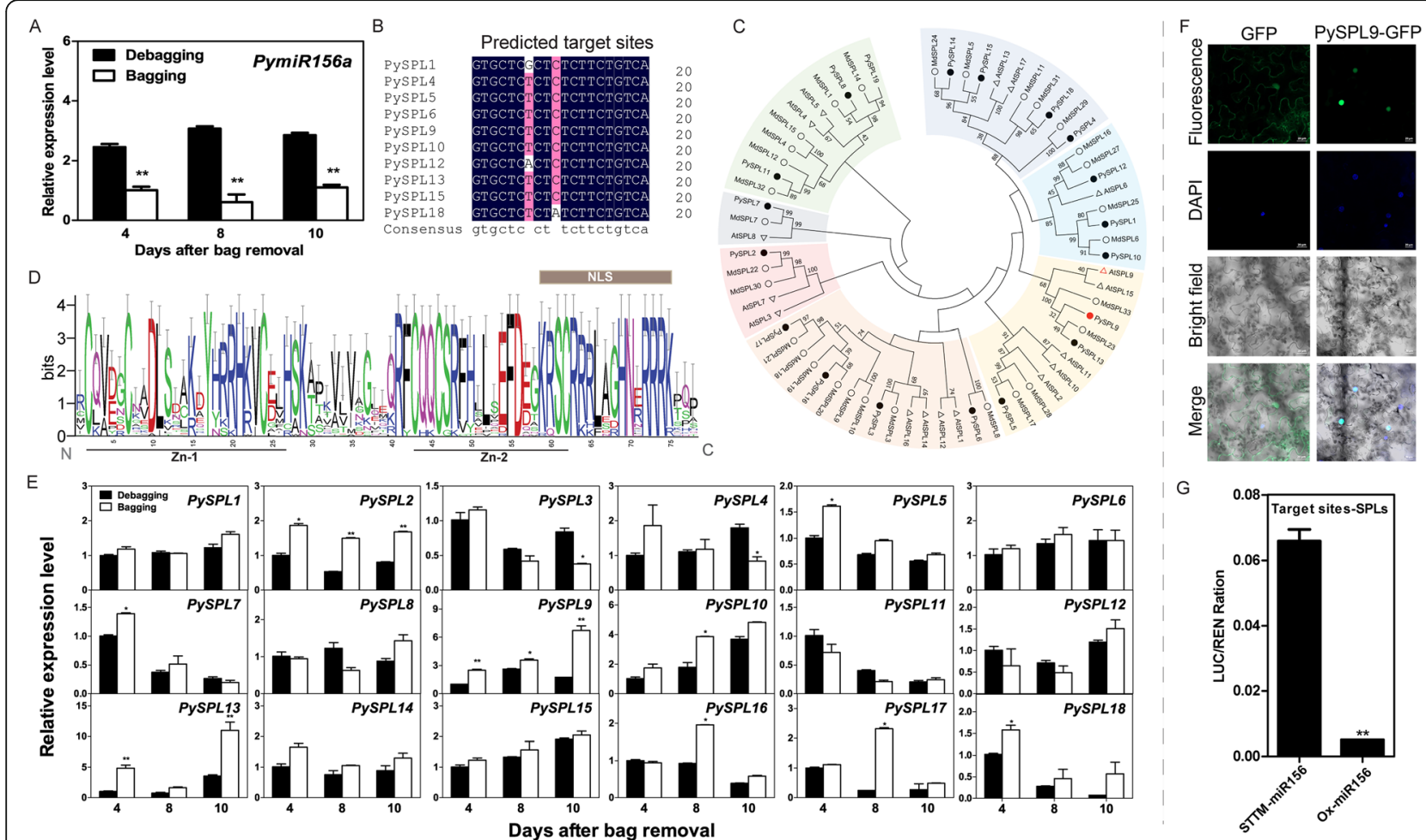

Fig. 3 The expression profile of PymiR156a and its targets analysis. A The expression profile of PymiR156a in bagged and debagged fruits skins. B Predicted target sites on PySPLS of PymiR156a. C Phylogenetic analysis of SPLs from pear, apple and Arabidopsis thaliana. D Sequence logo of the SBP domain of PySPL proteins (by weblogo.berkeley.edu). The conserved zinc finger structures (Zn-1 and Zn-2) and the nuclear localization signal (NLS) are indicated. E Expression profile analysis of PySPLS. F Subcellular localization of PySPL9 protein. G Verification of cleavage events of PymiR156a on PySPL

accumulation in PymiR156a overexpression Arabidopsis seedlings, which promoted the cotyledon changed into red (Fig. 4C). Furthermore, the anthocyanin content determination of apple calli, inoculated pear skin and Arabidopsis seedling confirmed the accumulation of anthocyanin caused by PymiR156a (Fig. 4D) These results suggested that PymiR156a plays a positive role in anthocyanin biosynthesis.

To further illustrate the regulation between PymiR156a and PySPL9, co-expression PymiR156a and PySPL9, by mixing them using agrobacterium-mediated transformation in "Zaosu" pear were conducted. We observed that overexpression of PymiR156a alone promoted anthocyanin accumulation (Fig. 4), but not when co-expressed with PymiR156a and excessive PySPL9 (Fig. S5), which demonstrated that the effect of PymiR156a overexpression on pigment accumulation was blocked by increasing the abundance of PySPL9 in the fruit skin.

\section{Interaction between PymiR156a-targeted PySPL9 and PyMYB114/PyMYB10}

To explore the role of PymiR156a-targeted PySPL9 (homologous to Arabidopsis AtSPL9) during light- induced anthocyanin biosynthesis in pear skin, PySPL9 protein expression vectors were constructed in pGBKT7, and PyMYB114 and PyMYB10 were fused to pGADT7 to test interactions using the yeast two-hybrid assay (with $\mathrm{Y} 2 \mathrm{H}$ Gold Yeast Strain). Our results indicated that PySPL9 interacted with both PyMYB114 and PyMYB10 (Fig. 5A). $\beta$-galactosidase assays (o-Nitrophenyl- $\beta$-D-galactoside, ONPG) were carried out to quantify and compare the strength of this protein-protein interaction. No significant difference in interaction intensity between PySPL9-PyMYB114 and PySPL9PyMYB10 protein was observed (Fig. 5B), indicating similar stability of these two SPL-MYB protein complexes. The Firefly Luciferase Complementation Imaging (LCI) assay further confirmed the interaction of PySPL9-PyMYB114 and PySPL9-PyMYB10 (Fig. 5C).

In addition, we also compared the expression patterns of the complex members in bagged and debagged treatment, and found that the expression levels of PyERF3, PybHLH3 and PyWD40 in the skins from bagged fruits were relatively low (Fig. S6). Results are consistant with up-regulation of PyPIF5 

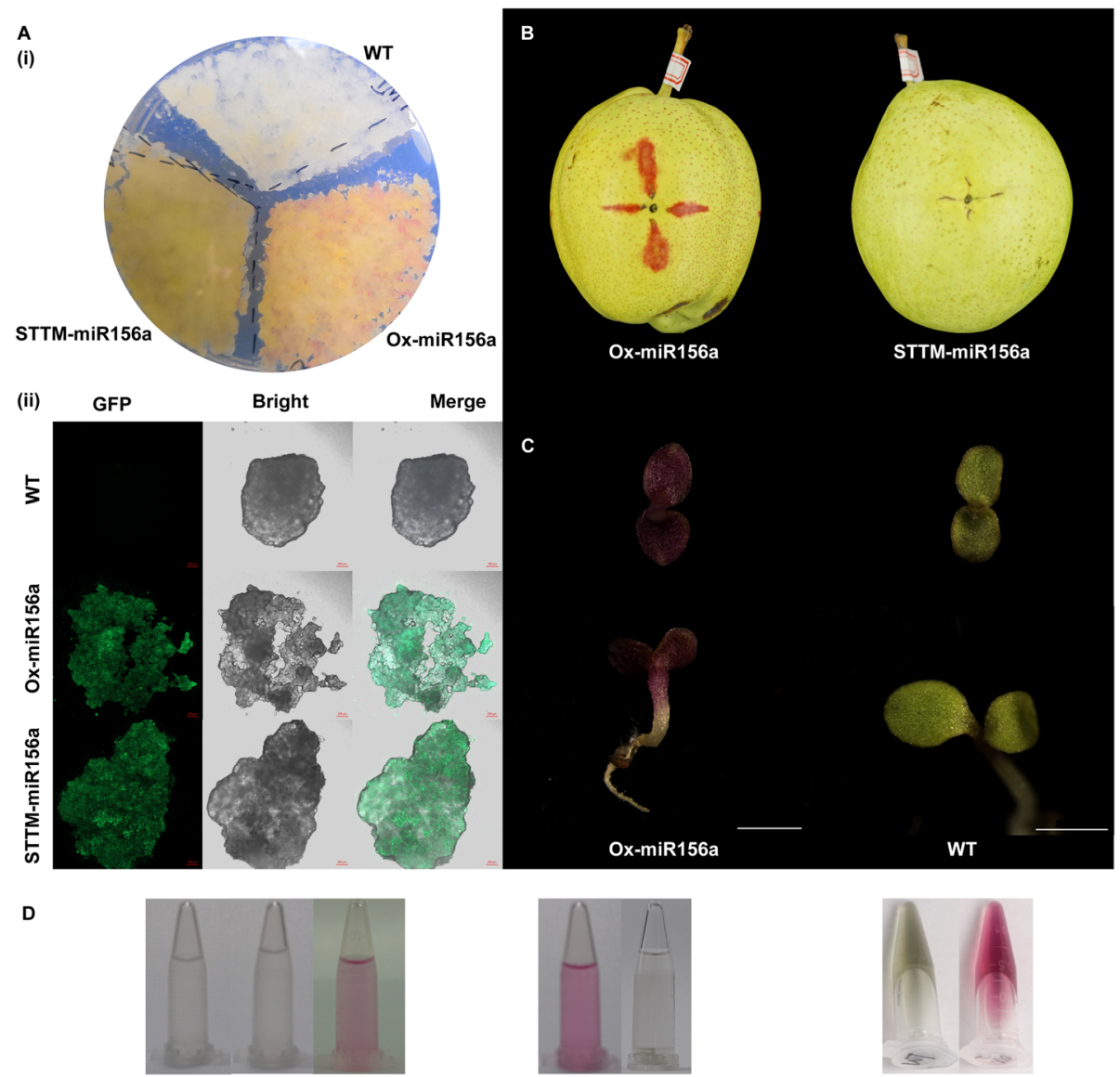

Merge
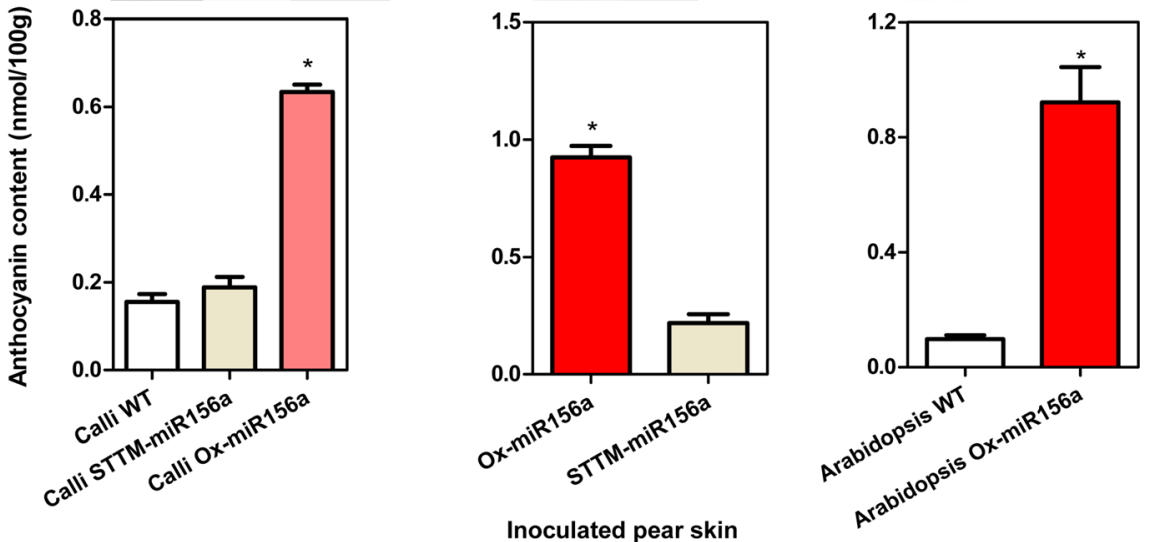

Fig. 4 Overexpression and suppression of PymiR156a. A Overexpression and suppression of PymiR156a in apple calli (i), and Green fluorescence detection (ii). B Overexpression and suppression of PymiR156a in Cv. "Zaosu". C Overexpression of PymiR156a in Arabidopsis seedling. D Anthocyanin content in apple calli, inoculated pear skin and Arabidopsis seedling (nmol/100 mg fresh weight)

which promotes the accumulation of PySPL9 by suppression the expression of PymiR156a. In debagged fruits there is low expression of PyPIF5 weakening its inhibitory effect on PymiR156a which would result in degradation of the target PySPL9. Transcripts of other complex members in the anthocyanin regulatory complex are upregulated to elevate expression of downstream anthocyanin related genes.

\section{Discussion}

The PyPIF5-PymiR156a-PySPL9-MYB114/10 module regulates light-induced anthocyanin biosynthesis in pear Light is an important environmental factor that affects the growth and development of plants. The complex and sophisticated systems for sensing and response to light signals have been the target of long-term evolution (Chory and Wu 2001). PIFs, which play a central role by 


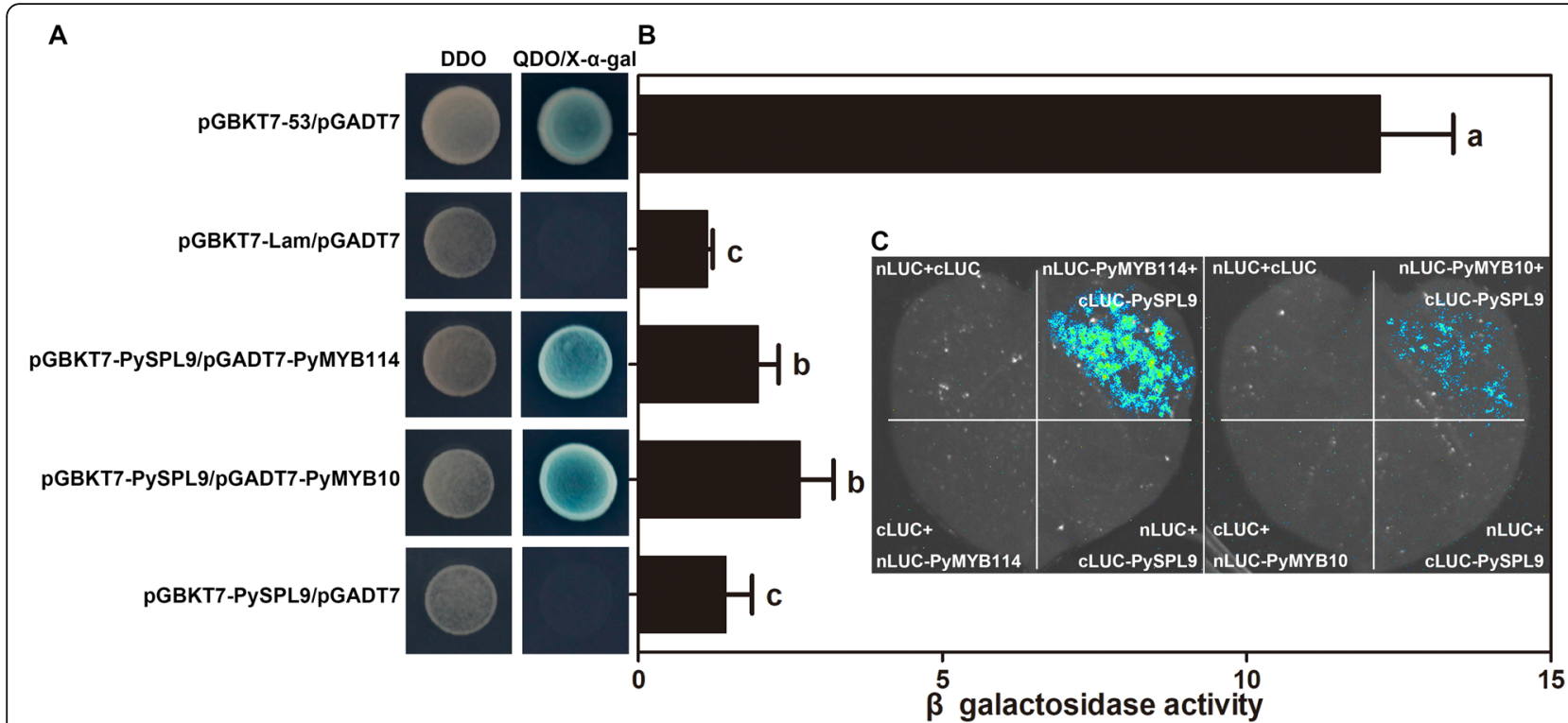

Fig. 5 The interaction between PymiR156a-targeted PySPL9 and PyMYB114/10. A The interaction between PySPL9 and PyMYB114/10 verified by yeast two-hybrid assays. DDO: double dropout SD medium (lacking leucine and tryptophan). QDO/X-a-gal: quadruple dropout SD medium (lacking adenine, histidine, leucine and tryptophan) containing X-a-gal. B Detection of $\beta$ galactosidase activity. C Firefly Luciferase Complementation Imaging (LCl) assay for protein-protein interactions

activating or repressing expression of downstream genes in light signaling during plant photomorphogenesis, work as a cellular signaling hub that integrates multiple signals to coordinate transcriptional networks (Shin et al. 2009; Leivar and Quail 2011; Pfeiffer et al. 2014). While, there are more than one PIFs among the differentially expressed genes (DEGs) in transcriptome data from bagged and debagged pear. A gene annotated as PIF3 is up-regulated in the debagged fruit compared to the fruit still in the bag. As previous studies have shown that PIF3 act as a positive regulator during anthocyanin accumulation (Shin et al. 2007), and we got a similar result in our experiments (Fig. S7). Therefore, we focused on the role of down-regulated PyPIF5 to discover and reveal differential regulatory mechanisms.

Some of these PIF-regulated networks include embryo development (Oh et al. 2004), seed germination (Leivar and Quail 2011; Oh et al. 2004), hypocotyl elongation (Monte et al. 2004), chlorophyll biosynthesis (Shin et al. 2009; Monte et al. 2004), shade avoidance (Xie et al. 2017; Nozue et al. 2011), and the circadian clock (Fujimori et al. 2004). In previous studies, it has been shown that PIF regulates shade avoidance by affecting AtmiR156 in Arabidopsis (Xie et al. 2017). However, to date there has been no evidence to support that PIFs act as upstream regulators of miRNA involved in anthocyanin biosynthesis.

To investigate potential regulators, we performed cisacting regulatory elements analysis of PymiR156a promoter. Multiple PIF-binding sites were found in
PymiR156a promoter. PyPIF5 was identified based on differential expression analysis, while dual-luciferase assays and EMSA results indicated that PyPIF5 negatively regulates PymiR156a expression. Based on these results, we propose a putative model (Fig. 6). We found that PyPIF5 acts as an upstream transcription factor that directly binds to the G-box motif in PymiR156a promoter and suppresses its expression in bagged fruits (in the absence of light), thus relieves the cleavage effect of PymiR156a on the target PySPL genes. PySPL then accumulates to form heterodimers with crucial members of the anthocyanin biosynthesis regulatory complexes (PyMYB114 and PyMYB10), thus blocking the regulation of downstream genes that encode anthocyanin biosynthesis enzymes. In contrast, PyPIF5 was significantly down-regulated when the fruits were re-exposed to natural light, and lost its inhibition of PymiR156a. Meanwhile, enough mature PymiR156a was produced and the targets were degraded thoroughly. In this way, the transcription complexes can be formed without being inhibited PySPL, thereby completing the regulation of anthocyanin biosynthesis genes (Fig. 6). From this data we reveal a direct regulatory link between two important regulators, upstream TFs and miRNAs, during lightinduced anthocyanin biosynthesis in pear.

Besides that, there were other TFs might participate in this process based on cis-acting element analysis of PymiR156a promoter. In addition to PIF-binding sites, GATA-binding sites (GATA-motif), BBX-binding sites (GT1-motif), and MYB-binding sites (I-box) were also 


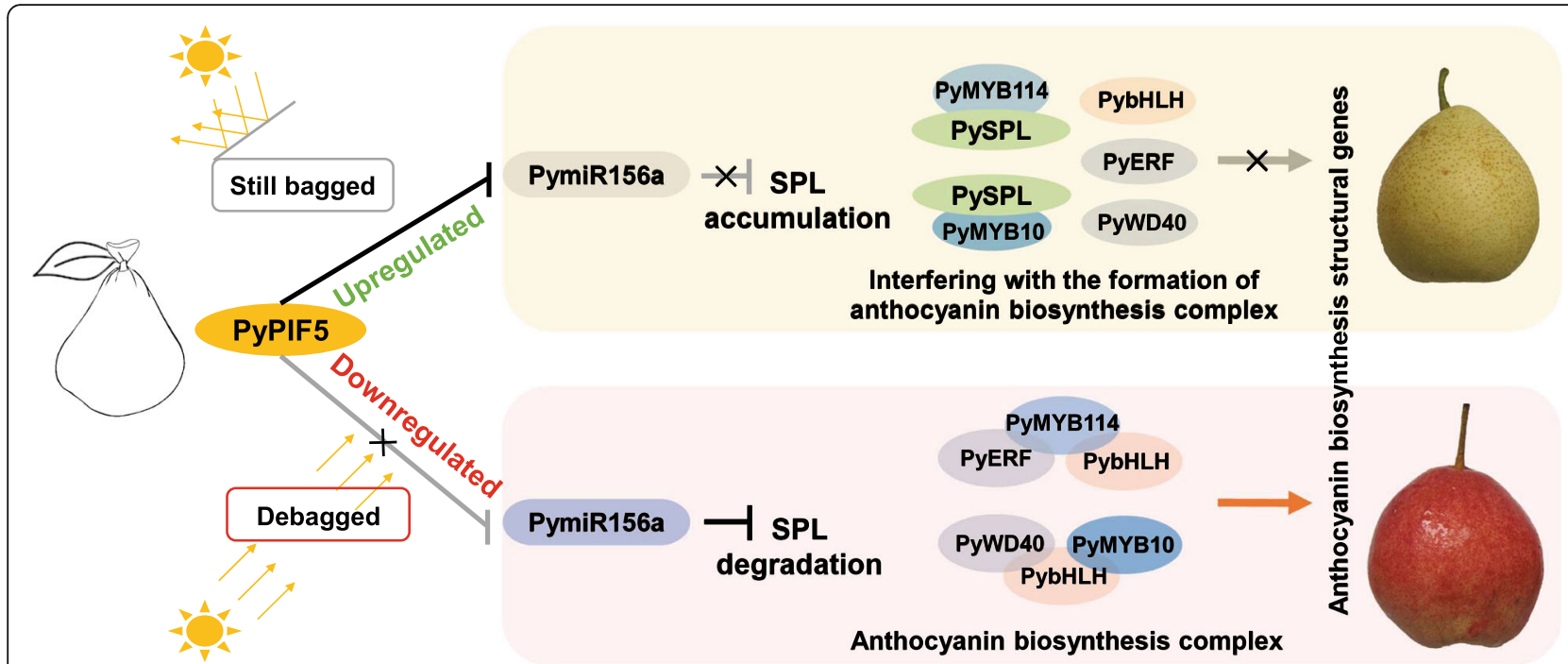

Fig. 6 A negative regulatory model of PyPIF5 on PymiR156a-mediated light-induced anthocyanin biosynthesis in pear. In the light PyPIF5 does not maintain repression of the expression of PymiR156a, therefore SPL MRNA is cleaved, and SPL proteins decline in the complex

found in the PymiR156a promoter. We investigated the transcription level of these TFs, and found that not all of these corresponding motif-binding TFs showed a similar expression pattern as PyPIF5 in bagged and debagged fruits (Fig. S8), which might be related to the complexity of light-induced anthocyanin regulation. In the current research we are not yet sure whether these TFs can bind to PymiR156a promoter and play regulatory effect, which needs further research. Considering the results of previous studies, other regulatory mechanisms (proteinprotein interaction, DNA-protein interaction, other miRNA-target interaction, and epigenetic regulation etc.) also contribute significantly in the late anthocyanin biosynthesis and the final ripening stage. This might be the reason for the inconsistency of different expression PyPIF5 and significant different expression PymiR156a at the 8th day. Therefore, we do not exclude the possibility that other TFs or regulatory mechanism have a regulatory effect on PymiR156a.

In summary, even though some progress has been made in the study of the regulation mechanism of light-induced anthocyanin biosynthesis for plant species, it remains to be determined how light response factor play a regulatory role during miRNA-mediated light-induced anthocyanin accumulation. In present study, we perfected regulatory mechanism of PymiR156a affecting anthocyanin biosynthesis through target genes, and pioneered an alternative PIF5-miRNA-SPLMYB module in light-induced anthocyanin biosynthesis.

Heterodimers of PySPL9 with PyMYB114 or PyMYB10 may co-exist in bagged fruits

MiR156 is an important miRNA family that plays roles in plant growth and development, metabolism regulation and stress response, and exerts biological functions through modulating target SPL genes (Cui et al. 2014; Cui et al. 2020; Gou et al. 2011; Miao et al. 2019). The SPL family is a plant specific transcription factor class with multiple functions. Gene family analysis of SPLs has been completed in diverse plant species, such as Arabidopsis (Cardon et al. 1999), apple (Li et al. 2013), grapevine (Wang et al. 2010), tomato (Salinas et al. 2012), rice (Xie et al. 2006) and cotton (Cai et al. 2018). Some members have been reported to be involved in anthocyanin biosynthesis in Arabidopsis (Gou et al. 2011), apple (Yang et al. 2019), peach (Zhou et al. 2015) and pear (Qian et al. 2017). In the present study, we identified that PymiR156a was significantly upregulated, while several PySPLS (Take SPL9 as a typical representative) were significantly downregulated during lightinduced anthocyanin accumulation (Fig. 3D), and this was caused by cleavage events driven by PymiR156 on PySPL (Fig. 3G). In addition to PySPL9, PySPL13, which is also one of the predicted targets of PymiR156a, was also differentially expressed in Ox-miR156a inoculated red sector (Fig. S9), indicating the possible regulating function for PymiR156a-PySPL13. Comparatively, the PySPL13 showed significant differences in two stages, except 8 days after debagging (Fig. 3E). We deduced the function of PymiR156a targeting PySPL13 might involve in anthocyanin regulation, but not by activating PySPL9 in all stages.

Interestingly, the expression of SPL family members in bagged fruits was generally lower than that of debagged fruits. This was contrary to the expression trend seen in apple where SPL2 and SPL33 activated anthocyanin biosynthesis (Yang et al. 2019). According to phylogenetic 
and qRT-PCR analysis (Fig. 3), apple SPL2 and SPL33 were not in the same cluster, and the expression pattern of the pear homologous genes were different, suggesting different and diverse functions of the SPL family between species.

It was known that the formation of an ERF/MYB/ bHLH and MYB/bHLH/WD40 complex plays important roles in the anthocyanin biosynthesis in pear (Yao et al. 2017; Qian et al. 2017). We revealed that PySPL9 (a close homolog of $A$. thaliana AtSPL9) interacted with PyMYB114 or PyMYB10 (Fig. 5A), which are the central members of the anthocyanin biosynthesis regulatory complexes (Feng et al. 2010; Yao et al. 2017). These potentially form two independent heterodimers. In addition, we also evaluated the protein interaction strength in these two heterodimers by use of the $\beta$ galactosidase activity. No significant difference was observed between the two heterodimers, which indicated that they could coexist to function redundantly.

As two important transcriptional regulators, we have proved in vitro that PyMYB114 and PyMYB10 interact with the target PySPL of PymiR156a and proposed possible regulatory models, whether there is any interaction in pear requires further in vivo experimental verification.

We performed small RNA and transcriptome sequencing analysis with bagged and debagged pear fruits. The integrated analysis of DE-miRNAs and DEGs combined with experimental results revealed that a specific PIF, PyPIF5 works as a negative regulator of anthocyanin biosynthesis in the absence of light. PyPIF5 directly binds to the G-box motif in the promoter of PymiR156a to suppress its expression, which relieves the cleavage effect of PymiR156a on target PySPL9. The accumulated PySPL9 then forms heterodimers with PyMYB114 or PyMYB10 proteins. The regulation of MYBs related complex to downstream anthocyanin-related biosynthesis genes is therefore blocked. Our results complement the study of upstream regulation of miRNA towards understanding of the miRNA-mediated light-induced anthocyanin biosynthesis regulation network.

\section{Methods}

\section{Plant materials and treatments}

Red Chinese sand pear (cultivar "Yunhongyihao") fruits were obtained from Yunnan Academy of Agricultural Sciences in Kunming, Yunnan, China. We bagged fruitlets at 35 days after full bloom with double-layered yellow-black paper bags [Kobayashi (Qingdao) Co., Ltd., China]. Half of the fruits were re-exposed to sunlight after the bags were removed at 10 days before harvest, and fruits were randomly sampled at 4 (D1), 8 (D2), and 10 (D3) days after bag removal (DABR). The fruits that remained bagged served as control samples (B1, B2 and B3). The skin from 10 individual fruits were pooled, instantly frozen in liquid nitrogen, and stored at $-80^{\circ} \mathrm{C}$ for assays.

\section{Small-RNA sequencing and data analysis}

A total amount of $3 \mu \mathrm{g}$ total RNA from B1, B2, B3, D1, D2 and D3 (three biological replicates in per sample) was used for small RNA library construction. Sequencing libraries were constructed by NEBNext ${ }^{\circ}$ Multiplex Small RNA Library Prep Set for Illumina ${ }^{\circ}$ (NEB, USA). After library quality assessment and cluster generation, the qualified libraries were sequenced by an Illumina Hiseq 2500 platform with $50 \mathrm{bp}$ single-end reads. After filtering, the remaining clean reads were used to search against miRBase database (Released 21, http://www. mirbase.org/) or align miRNA sequences in miRBase against reference genome for pear miRNAs identification. The available software miREvo (Wen et al. 2012) and mirdeep2 (Friedlander et al. 2011) were integrated to predict novel miRNA. psRobot_tar in psRobot (Wu et al. 2012) was used for the target gene prediction of miRNAs. DESeq R package (1.8.3) was used for differential expression analysis.

\section{RNA-sequencing and data analysis}

A total amount of $1 \mu \mathrm{g}$ RNA from B1, B2, B3, D1, D2 and D3 (three biological replicates in per sample) was used for the RNA sample preparations. Sequencing libraries were generated using NEBNext ${ }^{\circ}$ Ultra $^{\mathrm{Tw}}$ RNA Library Prep Kit for Illumina ${ }^{\circ}$ (NEB, USA). Illumina Hiseq platform was used for sequencing and $125 \mathrm{bp} / 150 \mathrm{bp}$ paired-end reads were generated. Clean reads were obtained after filtering and removing. The Q20, Q30, GCcontent, and also the duplication levels were calculated. All subsequent analyses were based on clean data with high quality. DESeq R package (1.18.0) was used for differential expression analysis, GO and KEGG enrichment analysis of DEGs were also performed.

\section{Expression profiles analysis}

Total RNA was extracted using a modified CTAB method (Porebski et al. 1997). The total RNA sample was treated with DNase I to remove any contaminating genomic DNA, and then quantified.

For miRNA qRT-PCR (Quantitative reverse transcription PCR) analyses, miRNA first-Strand was synthesized using the Mir- $\mathrm{X}^{\mathrm{m}}$ miRNA First-Strand Synthesis and SYBR $^{\circ}$ qRT-PCR Kit according to the instructions (638,313, Takara Biotechnology Co., Ltd). For target mRNA qRT-PCR analyses, first-strand cDNA was synthesized using TransScript ${ }^{\circ}$ One-Step gDNA Removal and cDNA Synthesis SuperMix (AT311, Transgene, Beijing, China) (Pei et al. 2020).

The qPCR experiment was performed on LightCycler 480 Real-Time PCR instrument (Roche Diagnostics ${ }^{\circ}$, 
Basel, Switzerland). All reactions were performed with three replications, and U6 snRNA and PyTUB were respectively used as the internal control for miRNA and mRNA. Relative expression level of each gene was calculated using the $2^{-\Delta \Delta C p}$ algorithm. The $\mathrm{qPCR}$ primers are listed in Supplementary Table S2.

\section{Assay of promoter activity and GUS staining}

The expression vector consisting of the $\beta$-glucuronidase reporter gene (GUS) coding sequence driven by PymiR156a promoter was constructed by inserting the promoter into Hind III and EcoR I cloning sites of pCAMBIA0390::GUS vector by using the corresponding primers (Table S2). After confirmation by sequencing, the recombinant construct was transformed into Agrobacterium tumefaciens strain GV3101 for transient expression in Nicotiana benthamiana leaves through vacuum infiltration (Santos-Rosa et al. 2008). Then, the transformed leaves were cultivated at $25^{\circ} \mathrm{C}$ for $24 \mathrm{~h}$.

GUSBlue KIT (GT0391 \& GT0392, Huayueyang Biotech Co., Ltd., Beijing, China) was used for histochemical GUS staining according to the instruction. Following staining (after $12 \mathrm{~h}$ of staining), the transformed leaves were washed with a graded ethanol series (70-90\%) to wipe off chlorophyll until they turn transparent. Expression of pro-PymiR156a::GUS was observed and photographed using a digital camera.

\section{PyPIF5-his fusion protein expression and purification}

The open reading frames of PyPIF5 were cloned into the pColdTF vector, which contained a Histidine (His) tag sequence, and the recombinant vector (pCold-PyPIF5) was then transformed into Escherichia coli BL21. The BL21 bacteria contained pCold-PyPIF5 was cultured at $37^{\circ} \mathrm{C}(200 \mathrm{rpm})$ until $\mathrm{OD}_{600} \sim 0.6$. After cooling, the liquid culture was subsequently treated with $1 \mathrm{mM}$ $(100 \mu \mathrm{L} / 100 \mathrm{~mL})$ isopropyl $\beta$-D-1-thiogalactopyranoside (IPTG) to induce the PyPIF5-His fusion protein production (at $16^{\circ} \mathrm{C}, 150 \mathrm{rpm}$ for $24 \mathrm{~h}$ ). The fusion protein was released from the cells by sonication and further purified with Ni-NTA His Bind Resin (Sangon Biotech. Co., Ltd., Shanghai, China). After elution with different concentration gradients of imidazole, the proteins were collected and detected by SDS-PAGE (Sodium dodecyl sulfate polyacrylamide gel electrophoresis).

\section{EMSA}

EMSA was performed using biotin-labeled probes and the LightShift Chemiluminescent EMSA Kit (GS009, Beyotime, Shanghai, China). The PymiR156a promoter sequence containing G-box were used to generate the biotin-labeled (Bio-probe) and unlabeled probes (Coldprobe). In addition, biotin-labeled mutate-probe (BioMut-probe) was generated by mutating recognition site
(G-box motif) for competition analysis. Briefly, $500 \mathrm{ng}$ of PyPIF5-His protein was incubated together with $20 \mathrm{nM}$ biotin-labeled probes in $20 \mu \mathrm{L}$ reaction mixtures (with $4 \mu \mathrm{L} 5 \times \mathrm{EMSA} /$ Gel-Shift and Nuclease-Free Water for supplement the reaction volume) for $20 \mathrm{~min}$ at room temperature $\left(\sim 25^{\circ} \mathrm{C}\right)$. Cold-probe or Mut-probe was added for the competition reactions.

\section{Overexpression and suppression vector construction for PymiR156a and agrobacterium-mediated transformations} To generate PymiR156a overexpression vector, a 300-bp stem-loop fragment of PymiR156 precursor was amplified by PCR from the pear genomic DNA and inserted into $p E A S Y^{\circ}$-Blunt cloning vector (CB101, Transgene, Beijing, China). After monoclonal sequencing, the stemloop fragment was inserted into the GFP vector, which carries green fluorescent protein and the selectable marker gene hygromycin phosphotransferase $(h p h)$.

For PymiR156a suppression vector, the short tandem target mimic (STTM) construction was referred to, as described by Tang et al. (2012). The STTM structure was constructed by PCR, and inserted into an intermediate vector $\left(p E A S Y^{\circ}\right.$-Blunt cloning vector, Transgene, Beijing, China), then introduced into the GFP vector through $\mathrm{BamH} \mathrm{I}$ and $\mathrm{Xba}$ I sites. The final expression vector GFP-Ox-PymiR156, GFP-STTM-PymiR156 (Fig. S10) were transferred into Agrobacterium tumefaciens strain GV3101 using the freezing/heat shock method. And the suppression and overexpression vector of PyPIF5 were also constructed by homologous recombination.

The transient transformation of pear skin and apple calli was as previously reported (Yao et al. 2017; An et al. 2017). Arabidopsis thaliana was used for Agrobacterium-mediated transformation following the procedure described by Clough and Bent (2018). Total anthocyanin content of the extracts was determined by spectrophotometric method (Vieira et al. 2018).

\section{Dual-luciferase reporter assay}

PymiR156a targets were predicted by using the psRNATarget server (http://plantgrn.noble.org/psRNATarget/) (Dai and Zhao 2011). According to the prediction, the pGreenII Dual-Luciferase miRNA Target Expression Vector (Xue et al. 2019) derived from pGreenII 0800LUC (Hellens et al. 2005) was used to quantitatively evaluate miRNA activity. For this evaluation, GFP-OxPymiR156, GFP-STTM-PymiR156 were used as effector, the reporter vector construction was as previously described by Xue et al. (2019), the predicted PymiR156a target and its flanking sequence ( 100-bp upstream and downstream of target sequence) was amplified and inserted to 3 ' terminal of the LUC gene (Fig. S10) by 
using ClonExpress ${ }^{\oplus}$ II One Step Cloning Kit (C112, Vazyme Biotech Co., Ltd., Nanjing, China).

Nicotiana benthamiana, which used for dual-luciferase reporter assay, were grown in a greenhouse with $16 \mathrm{~h}$ light at an average daily temperature of $25^{\circ} \mathrm{C}$. Four- to six-week-old Nicotiana benthamiana plants were infiltrated at the lower epidermal side of the leaves with needleless syringe. At least six leaves were infiltrated by one combination. Three days after infiltration, the leaves were used for dual-luciferase assay by the DualLuciferase Reporter Assay System (E1910, Promega Corporation, Madison, USA).

\section{Yeast two-hybrid and $\mathrm{LCl}$ assay}

For yeast two-hybrid assay, the coding regions of PyMYB114 and PyMYB10, PySPL9 were PCR amplified from cDNAs and ligated to the pGADT7 and pGBKT7 vectors (Clontech, USA) to generate pGADT7-PyMYB114 and pGADT7-PyMYB10, and pGBKT7-PySPL9, respectively. The constructs were cotransformed into the yeast strain Y2H Gold (Clontech, Saint-Germain-en-Laye, France). Yeast transformation and liquid assay were conducted as described in the Yeast Protocols Handbook (Clontech). Transformants were grown on proper dropout plates containing $\mathrm{X}$ - $\alpha$-gal (5-bromo-4-chloro-3-indolyl $\alpha$-D-galactopyranoside) for positive interactions verification.

LCI assays were performed as previously described (Chen et al. 63). Briefly, Agrobacterium strain GV3101 carrying nLUC (nLUC-PyMYB114 and nLUCPyMYB10) and cLUC (cLUC-PySPL9) construct combinations were infiltrated into tobacco leaves. LUC activities were detected using plant living imaging system (Princeton Instruments, USA).

\section{Supplementary Information}

The online version contains supplementary material available at https://doi. org/10.1186/s43897-021-00018-5.

Additional file 1 : Fig. S1. Functional enrichment and overlap of predicted targets and DEGs.

Additional file 2 : Fig. S2. Promoter analysis of PymiR156a in transient assay using GUS reporter gene.

Additional file $\mathbf{3}$ : Fig. S3. Sequence logo of the APB and $b H L H$ domain of PyPIF proteins.

Additional file 4 : Fig. S4. PyPIF5-His fusion protein and Probe sequence containing the G-box.

Additional file 5 : Fig. S5. Co-expression of PymiR156a and PYSPL9. Additional file $\mathbf{6}$ : Fig. S6. Expression pattern analysis of anthocyanin regulatory complex members.

Additional file $\mathbf{7}$ : Fig. S7. Expression pattern analysis of PyPIF3 in begged and debagged fruits.

Additional file 8 : Fig. S8. Heatmap analysis of TFs that binding to corresponding cis-acting elements in PymiR156a promoter.
Additional file 9 : Fig. S9. Expression analysis of PySPL9 and PySPL 13 in Ox-miR156a inoculated pear skin.

Additional file 10 : Fig. S10. Schematic representation of effector and reporter constructs used for dual-luciferase assays.

Additional file 11 : Table S1. Light-responsive cis-acting elements in promoter region of pre-PymiR156a.

Additional file 12 : Table S2. The primer sequences used in this study.

\section{Acknowledgements}

Not applicable.

\section{Authors' contributions}

WJ conceived and designed the experiments. LHN carried out the data analysis and conducted molecular experiments, and wrote the paper. SJ and SQ provided experimental materials, and performed bagging treatment and sample collection. LHN and SQ contributed equally to this work. WJ, ACA,

RVE, KL-W, and PMS designed gene function study, data analysis, and revised the manuscript. All authors have read and approved the final manuscript.

\section{Funding}

This work was supported by the National Natural Science Foundation of China (31820103012), the National Key Research and Development Program (2018YFD1000200), China Agriculture Research System of MOF and MARA, the Earmarked Fund for Jiangsu Agricultural Industry Technology System (JATS [2020]401).

\section{Availability of data and materials}

The datasets during and/or analyzed during the current study available from the corresponding author on reasonable request.

\section{Declarations}

Ethics approval and consent to participate

The red Chinese sand pear (cultivar "Yunhongyihao") fruits in current study were obtained from Yunnan Academy of Agricultural Sciences in Kunming, Yunnan, China. We are allowed to use them for scientific research.

Consent for publication

Not applicable.

\section{Competing interests}

The authors declare that they have no competing interests.

\section{Author details}

${ }^{1}$ College of Horticulture, State Key Laboratory of Crop Genetics and Germplasm Enhancement, Nanjing Agricultural University, Nanjing 210095, China. ${ }^{2}$ College of Horticulture and Plant Conservation, Henan University of Science and Technology, Luoyang 471023, China. ${ }^{3}$ Institute of Horticulture, Yunnan Academy of Agricultural Sciences, Kunming 650205, China. ${ }^{4}$ The New Zealand Institute for Plant \& Food Research Limited, Auckland, New Zealand. ${ }^{5}$ School of Biological Sciences, University of Auckland, Auckland, New Zealand.

Received: 13 February 2021 Accepted: 10 September 2021

Published online: 03 November 2021

References

An J, Qu F, Yao J, Wang X, You C, Wang X, et al. The bZIP transcription factor MdHY5 regulates anthocyanin accumulation and nitrate assimilation in apple. Hortic Res. 2017:4(1):17023. https://doi.org/10.1038/hortres.2017.23.

An J, Zhang X, You C, Bi S, Wang X, Hao Y. MdWRKY40 promotes woundinginduced anthocyanin biosynthesis in association with MdMYB1 and undergoes MdBT2-mediated degradation. New Phytol. 2019;224(1):380-95. https://doi.org/10.1111/nph.16008.

An XH, Tian Y, Chen KQ, Wang XF, Hao YJ. The apple WD40 protein MdTTG1 interacts with bHLH but not MYB proteins to regulate anthocyanin accumulation. J Plant Physiol. 2012;169(7):710-7. https://doi.org/10.1016/j. jplph.2012.01.015 
Azuma A, Yakushiji H, Koshita Y, Kobayashi S. Flavonoid biosynthesis-related genes in grape skin are differentially regulated by temperature and light conditions. Planta. 2012;236(4):1067-80. https://doi.org/10.1007/s00425-012-1 650-x.

Bai S, Tao R, Tang Y, Yin L, Teng Y. BBX16, a B-box protein, positively regulates light-induced anthocyanin accumulation by activating MYB10 in red pear. Plant Biotechnol J. 2019;17(10):1985-97. https://doi.org/10.1111/pbi.13114.

Cai CP, Guo WZ, Zhang BH. Genome-wide identification and characterization of SPL transcription factor family and their evolution and expression profiling analysis in cotton. Sci Rep. 2018;8(1):762. https://doi.org/10.1038/s41598-01 7-18673-4.

Cardon G, Hohmann S, Klein J, Nettesheim K, Saedler H, Huijser P. Molecular characterisation of the Arabidopsis SBP-box genes. Gene. 1999;237:91-104. https://doi.org/10.1016/S0378-1119(99)00308-X.

Chen H, Zou Y, Shang Y, Lin H, Wang Y, Cai R, Tang X, Zhou JM. Firefly luciferase complementation imaging assay for protein-protein interactions in plants. Plant Physiol. 2008;146(2):368-76. https://doi.org/10.1104/pp.107.111740.

Chory J, Wu D. Weaving the complex web of signal transduction. Plant Physiol. 2001;125(1):77-80. https://doi.org/10.1104/pp.125.1.77

Clough SJ, Bent AF. Floral dip: a simplified method for Agrobacterium mediated transformation of Arabidopsis thaliana. Plant J. 2018;16(6):735-43. https://doi. org/10.1046/j.1365-313x.1998.00343.x

Cui L, Zheng FY, Wang JF, Zhang CL, Xiao FM, Ye J, et al. miR156a-targeted SBPbox transcription factor SISPL13 regulates inflorescence morphogenesis by directly activating SFT in tomato. Plant Biotechnol J. 2020;18(8):1670-82. https://doi.org/10.1111/pbi.13331.

Cui LG, Shan JX, Shi M, Gao JP, Lin HX. The miR156-SPL9-DFR pathway coordinates the relationship between development and abiotic stress tolerance in plants. Plant J. 2014;80(6):1108-17. https://doi.org/10.1111/ tpj.12712.

Dai X, Zhao PX. psRNATarget: a plant small RNA target analysis server. Nucleic Acids Res. 2011;39(suppl_2):W155-9. https://doi.org/10.1093/nar/gkr319.

Espley RV, Hellens RP, Putterill J, Stevenson DE, Kutty-Amma S, Allan AC. Red colouration in apple fruit is due to the activity of the MYB transcription factor, MdMYB10. Plant J. 2007:49(3):414-27. https://doi.org/10.1111/j.13 65-313X.2006.02964.X.

Feng F, Li M, Ma F, Cheng L. Phenylpropanoid metabolites and expression of key genes involved in anthocyanin biosynthesis in the shaded peel of apple fruit in response to sun exposure. Plant Physiol Bioch. 2013;69:54-61. https://doi. org/10.1016/j.plaphy.2013.04.020.

Feng SQ, Wang Y, Yang S, XU Y, Chen X. Anthocyanin biosynthesis in pears is regulated by a R2R3-MYB transcription factor PyMYB10. Planta. 2010;232(1): 245-55. https://doi.org/10.1007/s00425-010-1170-5.

Friedlander MR, Mackowiak SD, Li N, Chen W, Rajewsky N. miRDeep2 accurately identifies known and hundreds of novel microRNA genes in seven animal clades. Nucleic Acids Res. 2011;40(1):37-52. https://doi.org/10.1093/nar/ gkr688.

Fujimori T, Yamashino T, Kato T, Mizuno T. Circadian controlled basic/helix-loophelix factor, PIL6, implicated in light-signal transduction in Arabidopsis thaliana. Plant Cell Physiol. 2004;45(8):1078-86. https://doi.org/10.1093/pcp/ pch124.

Gou JY, Felippes FF, Liu CJ, Weigel D, Wang JW. Negative regulation of anthocyanin biosynthesis in Arabidopsis by a miR156-targeted SPL transcription factor. Plant Cell. 2011;23(4):1512-22. https://doi.org/10.1105/ tpc.111.084525.

Guo DL, Wang ZG, Li Q, Gu SC, Zhang GH, Yu YH. Hydrogen peroxide treatment promotes early ripening of Kyoho grape. Aust J Grape Wine R. 2019;25(1): 357-62. https://doi.org/10.1186/s12864-020-07180-y.

Hellens RP, Allan AC, Friel EN, Bolitho K, Grafton K, Templeton MD, Karunairetnam S, Gleave AP, Laing WA. Transient expression vectors for functional genomics, quantification of promoter activity and RNA silencing in plants. Plant Methods. 2005;1:13. https://doi.org/10.1186/1746-4811-1-13.

Jia X, Shen J, Liu H, Li F, Ding N, Gao C, et al. Small tandem target mimic-mediated blockage of microRNA858 induces anthocyanin accumulation in tomato. Planta. 2015;242(1):283-93. https://doi.org/10.1007/s00425-015-2305-5.

Jiang YH, Liu CH, Yan D, Wen XH, Liu YL, Wang HJ, et al. MdHB1 down-regulation activates anthocyanin biosynthesis in the white-fleshed apple cultivar 'Granny Smith'. J Exp Bot. 2017;68(5):1055-69. https://doi.org/10.1093/jxb/ erx029.

Leivar P, Quail PH. PIFs: pivotal components in a cellular signaling hub. Trends Plant Sci. 2011;16(1):19-28. https://doi.org/10.1016/j.tplants.2010.08.003.
Li C, Wu J, Hu K, Wei S, Sun H, Hu L, et al. PyWRKY26 and PybHLH3 cotargeted the PyMYB114 promoter to regulate anthocyanin biosynthesis and transport in red-skinned pears. Hortic Res. 2020;7(1):37. https://doi.org/10.1038/s41438020-0254-z.

Li J, Hou H, Li X, Xiang J, Yin X, Gao H, et al. Genome-wide identification and analysis of the SBP-box family genes in apple (Malus $\times$ domestica Borkh.). Plant Physiol Bioch. 2013;70:110-4. https://doi.org/10.1016/j.pla phy.2013.05.021.

Liu HN, Su J, Zhu YF, Yao GF, Allan AC, Ampomah-Dwamena C, et al. The involvement of PybZIPa in light-induced anthocyanin accumulation via the activation of PyUFGT through binding to tandem G-boxes in its promoter. Hortic Res. 2019;6(1):134. https://doi.org/10.1038/s41438-019-0217-4.

Liu XJ, An XH, Liu X, Hu DG, Wang XF, You CX, et al. MdSnRK1.1 interacts with MdJAZ18 to regulate sucrose-induced anthocyanin and proanthocyanidin accumulation in apple. J Exp Bot. 2017;68(11):2977-90. https://doi.org/10.1 093/jxb/erx150

Miao CB, Wang Z, Zhang L, Yao JJ, Hua K, Liu X, et al. The grain yield modulator miR156 regulates seed dormancy through the gibberellin pathway in rice. Nat Commun. 2019;10(1):3822. https://doi.org/10.1038/ s41467-019-11830-5.

Monte E, Tepperman JM, Al-Sady B, Kaczorowski KA, Alonso JM, Ecker JR, et al. The phytochrome-interacting transcription factor, PIF3, acts early, selectively, and positively in light-induced chloroplast development. Proc Natl Acad Sci U S A. 2004;101(46):16091-8. https://doi.org/10.1073/pnas.0407107101.

Nozue K, Harmer SL, Maloof JN. Genomic analysis of circadian clock-, light-, and growth-correlated genes reveals PHYTOCHROME-INTERACTING FACTOR5 as a modulator of auxin signaling in Arabidopsis. Plant Physiol. 2011;156(1):357-72. https://doi.org/10.1104/pp.111.172684.

Oh E, Kim J, Park E, Kim Jl, Kang C, Choi G. PIL5, a phytochrome-interacting basic helix-loop-helix protein, is a key negative regulator of seed germination in Arabidopsis thaliana. Plant Cell. 2004;16(11):3045-58. https://doi.org/10.1105/ tpc.104.025163.

Pei MS, Cao SH, Wu L, Wang GM, Zhang SL. Comparative transcriptome analyses of fruit development among pears, peaches, and strawberries provide new insights into single sigmoid patterns. BMC Plant Biol. 2020;20(1):108. https:// doi.org/10.1186/s12870-020-2317-6.

Peng Z, Tian J, Luo R, Kang Y, Lu Y, Hu Y, et al. MiR399d and epigenetic modification comodulate anthocyanin accumulation in Malus leaves suffering from phosphorus deficiency. Plant Cell Environ. 2020;43(5):1148-59. https://doi.org/10.1111/pce.13697.

Pfeiffer A, Shi H, Tepperman JM, Zhang Y, Quail PH. Combinatorial complexity in a transcriptionally centered signaling hub in Arabidopsis. Mol Plant. 2014; 7(11):1598-618. https://doi.org/10.1093/mp/ssu087.

Porebski S, Bailey LG, Baum BR. Modification of a CTAB DNA extraction protocol for plants containing high polysaccharide and polyphenol components. Plant Mol Biol Rep. 1997;15(1):8-15. https://doi.org/10.1007/BF02772108.

Qian M, Ni J, Niu Q, Bai S, Bao L, Li J, et al. Response of miR156-SPL module during the red peel coloration of bagging-treated Chinese sand pear (Pyrus pyrifolia Nakai). Front Physiol. 2017;8:550. https://doi.org/10.3389/fphys.2017.00550.

Quattrocchio F, Wing JF, Leppen H, Mol J, Koes RE. Regulatory genes controlling anthocyanin pigmentation are functionally conserved among plant species and have distinct sets of target genes. Plant Cell. 1993;5(11):1497-512. https://doi.org/10.1105/tpc.5.11.1497.

Salinas M, Xing S, Höhmann S, Berndtgen R, Huijser P. Genomic organization, phylogenetic comparison and differential expression of the SBP-box family of transcription factors in tomato. Planta. 2012;235(6):1171-84. https://doi.org/1 0.1007/s00425-011-1565-y.

Santos-Rosa M, Poutaraud A, Merdinoglu D, Mestre P. Development of a transient expression system in grapevine via agro-infiltration. Plant Cell Rep. 2008;27(6): 1053-63. https://doi.org/10.1007/s00299-008-0531-z.

Shin J, Kim K, Kang H, Zulfugarov IS, Bae G, Lee CH, et al. Phytochromes promote seedling light responses by inhibiting four negatively-acting phytochromeinteracting factors. Proc Natl Acad Sci U S A. 2009;106(18):7660-5. https://doi. org/10.1073/pnas.0812219106

Shin J, Park E, Choi G. PIF3 regulates anthocyanin biosynthesis in an HY5dependent manner with both factors directly binding anthocyanin biosynthetic gene promoters in Arabidopsis. Plant J. 2007:49(6):981-94. https://doi.org/10.1111/j.1365-313X.2006.03021.x.

Tang G, Yan J, Gu Y, Qiao M, Fan R, Mao Y, et al. Construction of short tandem target mimic (STTM) to block the functions of plant and animal microRNAs. Methods. 2012;58(2):118-25. https://doi.org/10.1016/j.ymeth.2012.10.006. 
Toledo-Ortiz G, Huq E, Quail PH. The Arabidopsis basic helix-loop-helix transcription factor family. Plant Cell. 2003;15(8):1749-70. https://doi.org/10.11 05/tpc.013839.

Vieira LM, Marinho LMG, Rocha JCG, Barros FAR, Stringheta PC. Chromatic analysis for predicting anthocyanin content in fruits and vegetables. Food Sci Technol. 2018;39(2):415-22. https://doi.org/10.1590/fst.32517.

Wang Y, Hu Z, Yang Y, Chen X, Chen G. Genome-wide identification, phylogeny, and expression analysis of the SBP-box gene family in grapevine. Russ J Plant Physl. 2010;57(2):273-82. https://doi.org/10.1134/S1021443710020160.

Wen M, Shen Y, Shi S, Tang T. miREvo: An integrative microRNA evolutionary analysis platform for next-generation sequencing experiments. BMC Bioinformatics. 2012;13:140. https://doi.org/10.1186/1471-2105-13-140.

Wu HJ, Ma YK, Chen T, Wang M, Wang XJ. PsRobot: a web-based plant small RNA meta-analysis toolbox. Nucleic Acids Res. 2012;40(W1):W22-8. https://doi. org/10.1093/nar/gks554.

Xie K, Wu C, Xiong L. Genomic organization, differential expression, and interaction of SQUAMOSA promoter-binding-like transcription factors and microRNA156 in rice. Plant Physiol. 2006;142(1):280-93. https://doi.org/10.11 04/pp.106.084475.

Xie YR, Liu Y, Wang H, Ma XJ, Wang BB, Wu GX, et al. Phytochrome-interacting factors directly suppress MIR156 expression to enhance shade-avoidance syndrome in Arabidopsis. Nat Commun. 2017;8(1):348. https://doi.org/10.103 8/s41467-017-00404-y.

Xu D, Jiang Y, Li J, Lin F, Holm M, Deng X. BBX21, an Arabidopsis B-box protein, directly activates HY5 and is targeted by COP1 for 265 proteasome-mediated degradation. Proc Natl Acad Sci U S A. 2016;113(27):7655-60. https://doi. org/10.1073/pnas.1607687113.

Xu W, Dubos C, Lepiniec L. Transcriptional control of flavonoid biosynthesis by MYB-bHLH-WDR complexes. Trends Plant Sci. 2015;20(3):176-85. https://doi. org/10.1016/j.tplants.2014.12.001.

Xu W, Grain D, Le Gourrierec J, Harscoët E, Berger A, Jauvion V, et al. Regulation of flavonoid biosynthesis involves an unexpected complex transcriptional regulation of TT8 expression, in Arabidopsis. New Phytol. 2013;198(1):59-70, https://doi.org/10.1111/nph.12142.

Xue C, Yao JL, Qin MF, Zhang MY, Allan AC, Wang DF, Wu J. PbrmiR397a regulates lignification during stone cell development in pear fruit. Plant Biotechnol J. 2019:17(1):103-17. https://doi.org/10.1111/pbi.12950.

Yang F, Cai J, Yang Y, Liu Z. Overexpression of microRNA828 reduces anthocyanin accumulation in Arabidopsis. Plant Cell Tiss Org. 2013;15(2):15967. https://doi.org/10.1007/s11240-013-0349-4.

Yang T, Ma H, Zhang J, Wu T, Song T, Tian J, et al. Systematic identification of long noncoding RNAs expressed during light-induced anthocyanin accumulation in apple fruit. Plant J. 2019;100(3):572-90. https://doi.org/1 $0.1111 /$ tpj.14470

Yao GF, Ming ML, Andrew AC, Gu C, Li LT, Wu X, et al. Map-based cloning of the pear gene MYB114 identifies an interaction with other transcription factors to coordinately regulate fruit anthocyanin biosynthesis. Plant J. 2017;92(3):43751. https://doi.org/10.1111/tpj.13666

Yu YH, Bian L, Jiao ZL, Yu KK, Wan YT, Zhang GH, et al. Molecular cloning and characterization of a grapevine (Vitis vinifera L.) serotonin $\mathrm{N}$-acetyltransferase (VVSNAT2) gene involved in plant defense. BMC Genomics. 2019;20(1):1-13. https://doi.org/10.1186/s12864-019-6085-3.

Zhou H, Lin-Wang K, Wang H, Gu C, Dare AP, Espley RV, et al. Molecular genetics of blood-fleshed peach reveals activation of anthocyanin biosynthesis by NAC transcription factors. Plant J. 2015;82(1):105-21. https://doi.org/10.1111/ tpj.12792.

\section{Publisher's Note}

Springer Nature remains neutral with regard to jurisdictional claims in published maps and institutional affiliations.

Ready to submit your research? Choose BMC and benefit from:

- fast, convenient online submission

- thorough peer review by experienced researchers in your field

- rapid publication on acceptance

- support for research data, including large and complex data types

- gold Open Access which fosters wider collaboration and increased citations

- maximum visibility for your research: over $100 \mathrm{M}$ website views per year

At BMC, research is always in progress.

Learn more biomedcentral.com/submissions 